\title{
Thermal Expansion of the Cryoprotectant Cocktail DP6 Combined with Synthetic Ice Modulators in Presence and Absence of Biological Tissues
}

\author{
David P. Eisenberg ${ }^{2}$, Michael J. Taylor ${ }^{3}$, and Yoed Rabin ${ }^{2,1}$ \\ ${ }^{2}$ Biothermal Technology Laboratory, Department of Mechanical Engineering, Carnegie Mellon \\ University, Pittsburgh, PA 15213 \\ ${ }^{3}$ Cell and Tissue Systems, Inc., N. Charleston, SC
}

\section{Abstract}

This study explores physical effects associated with the application of cryopreservation via vitrification using a class of compounds which are defined here as synthetic ice modulators (SIMs). The general classification of SIMs includes molecules that modulate ice nucleation and growth, or possess properties of stabilizing the amorphous state, by virtue of their chemical structure and at concentrations that are not explained on a purely colligative basis. A subcategory of SIMs, referred to in the literature as synthetic ice blockers (SIBs), are compounds that interact directly with ice nuclei or crystals to modify their structure and/or rate of growth. The current study is part of an ongoing effort to characterize thermo-mechanical effects during vitrification, with emphasis on measuring the physical property of thermal expansion-the driving mechanism to thermo-mechanical stress. Materials under investigation are the cryoprotective agent (CPA) cocktail DP6 in combination with one of the following SIMs: $12 \%$ polyethylene glycol 400, $6 \%$ 1,3 cyclohexanediol, and 6\% 2,3 butanediol. Results are presented for the CPA-SIM cocktail in the absence and presence of bovine muscle and goat artery specimens. This study focuses on the upper part of the cryogenic temperature range, where the CPA behaves as a fluid for all practical applications. Results of this study indicate that the addition of SIMs to DP6 allows lower cooling rates to ensure vitrification and extends the range of measurements. It is demonstrated that the combination of SIM with DP6 increases the thermal expansion of the cocktail, with implications for the likelihood of fracture formation - the most dramatic outcome of thermo-mechanical stress.

\section{Keywords}

Thermal Expansion; Solid Mechanics; Vitrification; Synthetic Ice Blockers; Synthetic Ice Modulators

\section{INTRODUCTION}

The role of cryopreservation for tissue banking is undisputable, being the only practical alternative for long-term storage of high quality biomaterial. Techniques for successful

\footnotetext{
(c) 2012 Elsevier Inc. All rights reserved.

${ }^{1}$ Corresponding author: rabin@cmu.edu.

Publisher's Disclaimer: This is a PDF file of an unedited manuscript that has been accepted for publication. As a service to our customers we are providing this early version of the manuscript. The manuscript will undergo copyediting, typesetting, and review of the resulting proof before it is published in its final citable form. Please note that during the production process errors may be discovered which could affect the content, and all legal disclaimers that apply to the journal pertain.
} 
cryopreservation have been developed over the years for several tissue types. However, successful cryopreservation techniques are generally related to small specimens in the scalerange of cells to small-organized tissues, with the stem cells [12,29], corneas [11,40], and pancreatic islets [41] as examples. Larger-scale cryopreservation was accomplished only in cases where the mechanical functionality has a higher priority need than the recovery of biological functionality, with heart valves as an example [5,6]. While techniques of cryopreservation are continually developing, cryopreservation of organs and large-scale engineered tissue constructs is not yet available.

Classical cryopreservation, which makes use of low concentrations of cryoprotective agents (CPAs), such as dimethyl sulfoxide (DMSO), does a reasonable job of cell preservation by prevention of intracellular ice formation, but a poor job of tissue preservation. There have been several hypotheses on the mechanisms of freezing-induced injury based upon a variety of factors [19,21], but experience with mammalian tissues shows that the disadvantages of classical cryopreservation revolve primarily around ice formation $[22,23,37]$. The formation of extracellular ice in particular (generally regarded as innocuous for cells in suspension) is known to be hazardous to structured tissues and organs [13,14,22,23,37].

Vitrification is an alternative to conventional freezing of living biological materials with ubiquitous applications in cell, tissue, and organ storage [38]. In vitrification, the formation of ice crystals is suppressed by the presence of high-concentration CPA cocktails, which display an exponential increase in viscosity with the decreasing temperature. The typical time scale for crystal growth is proportional (but not linearly) to the level of viscosity of the material. When the CPA-permeated specimen is cooled fast enough, such that the typical time scale for crystallization is longer than the time period it takes to bring the specimen to low enough temperatures (i.e., where the viscosity is high enough), crystallization is practically avoided.

The temperature threshold below which the material is considered to have high enough viscosity to arrest crystal formation in any practical time scale is known as the glasstransition temperature, $T_{g}$ [30]. From solid-mechanics perspective, $T_{g}$ is commonly defined as the temperature at which the viscosity reaches the value of $10^{12} \mathrm{~Pa}-\mathrm{s}$. A typical viscosity value for CPA at room temperature is of the order of $10^{-3} \mathrm{~Pa}$-s, hence vitrification is associated with a dramatic viscosity increase in 15 orders of magnitude. Around $T_{g}$ the material is said to transition from a fluid-like to a solid-like behavior. From a thermodynamics perspective, differential scanning calorimetry (DSC) is often used to identify $T_{g}$, which is associated with internal energy changes (for alternative definitions of $T_{g}$ in this context see [30]). Either way, crystallization suppression is a kinetic effect and the outcome is path-dependent-it depends not only upon the final temperature but also upon the particular thermal history.

While vitrification is a relatively well understood physical process, first proposed for cryobiology applications by Luyet in 1937 [20], its application to biological systems is not without problems since the CPA concentration typically required to facilitate vitrification is very high and potentially toxic. To limit toxicity effects, it is necessary to use the least toxic CPA at the minimum concentration possible that still promotes glass formation $[8,9]$. Toxicity is not the only potentially devastating effect during vitrification, as the high cooling rates necessary for vitrification may give rise to thermo-mechanical stress [28]. Thermomechanical stress is the response of the material to volume changes associated with expansion or shrinkage of the material during cooling. When the level of stress exceeds the strength of the material, structural damage follows with fracture formation as its most dramatic outcome [27,31]. 
Compounds that influence the formation and growth of ice nuclei and crystals by various purported mechanisms are grouped in the current study under the generic term Synthetic Ice Modulators (SIMs). This general classification embraces several categories of molecules that have been shown to modulate ice formation and growth. For example, molecules such as 1,3 cyclohexanediol (1,3 CHD) that specifically attenuates the growth of ice crystals by virtue of its chemical structure have been referred to as synthetic ice blockers (SIB) [10,40]. In contrast, compounds such as synthetic polyvinyl alcohol have been suggested to be ice modulators primarily by inhibiting heterogeneous nucleation [42]. Such compounds should be considered as "anti-nucleating agents" as opposed to "ice blockers", but clearly fall within the general category of "ice modulators". The present study includes three contrasting SIMs: 1,3CHD represents the best published example of an SIB [10,40], and 2,3 butanediol and polyethylene glycol (PEG-400), which are included as compounds known to facilitate the stability of the amorphous state by virtue of their interactions with water (reviewed in [40]). In general, ice modulators have a greater effect on influencing ice nucleation and growth and stabilizing the amorphous state than accounted for on a pure colligative basis [40].

As with any cryoprotectant, the practical utility of SIMs in cryobiology represents a balance between physical and chemical properties and their interactions with living cells. It is generally recognized that toxicity is dependent upon concentration, and often temperature, irrespective of intrinsic physical properties. This study is focused purely on an intrinsic physical property (thermal expansion) of CPA cocktails containing a variety of SIMs without specific regard for potential toxicity other than choosing compounds that have been demonstrated to have some utility in biological applications. Nevertheless, the rational approach of reducing the inherent chemical toxicity of the CPA cocktails by reducing the total concentration of solutes while retaining desirable physical attributes is an underlying value of incorporating SIMs [40,41].

The combination of ordinary CPA cocktails with SIMs is further expected to reduce the rapid cooling rate necessary for vitrification, which is critical to scale-up cryopreservation for two primary reasons: (i) regardless of the cooling mechanism at the outer surface, the achievable cooling rate at the center of a bulky specimen is always lower-limited by principles of heat conduction through the tissue, and (ii) lower cooling rates often mean lower thermo-mechanical stress and diminishing risk to structural damage.

While the concept of SIM as a controlling agent of crystal growth is not new, very limited corresponding information is available in the literature of cryobiology. Of particular relevancy to the current study is a recent report on the application of the CPA cocktail DP6 combined with 1,3 cyclohexanediol (1,3-CHD) as an experimental SIB, which demonstrated significantly improved tendency to vitrification in the preservation of pancreatic islets [41]. That study showed higher islet viability post cryopreservation when compared with established reference vitrification solutions. More information on relevant SIMs is given in the Material and Methods below.

As a part of an ongoing effort to investigate thermo-mechanical effects in cryopreservation [15-19,26,27,32-35], the current study focuses on measuring key physical properties of the vitrifying specimen along the path of cryopreservation. In particular, thermal expansion is measured in the current study, which is the driving mechanism for thermo-mechanical stress. Since the vitrifying material changes behavior from fluid-like $[24,26]$ to solid-like $[15,16]$, two different experimental systems are required for mapping the effects of thermal expansion. The current study is focused on the upper part of the cryogenic temperature range, where the CPA-SIM cocktail behaves as a fluid. Beyond its key role in the formation 
of mechanical stress, thermal expansion can be used to study the extent of partial crystallization, when present, and the glass-transition temperature.

\section{MATERIALS AND METHODS}

\section{Experimental Setup}

A prototype of the apparatus for thermal expansion measurements used in the current study has been developed previously $[25,26]$ and is presented here in brief, for the completeness of presentation. With reference to Fig. 1, the experimental system consists of two main components: (i) a cooling chamber, which contains the specimen and is cooled to cryogenic temperatures, and (ii) a pressure tube, which includes a pressure transducer and contains air at room temperature. The two components are connected by means of a flexible tube, which is filled with the same CPA solution under investigation, but has a negligible volume compared with the volume of the cooling chamber. This is a closed system, where expansion of the specimen due to temperature changes causes pressure changes in the system. It follows that the measured quantity is the pressure, whereas the modeled parameter is the thermal expansion. In order to passively control the cooling rate, the cooling chamber is placed in a cylindrical insulation shell, which is immersed in liquid nitrogen. The shell thickness is designed to achieve the desired conditions for vitrification. T-type thermocouples are placed on the outside of the cooling chamber and at its center, in order to monitor the temperature and verify that it is close to uniform across the chamber. For the purpose of the current study, the cooling chamber has been modified from $11.1 \mathrm{ml}$ [26] to $3.0 \mathrm{ml}$, in order to increase the cooling rate. The thermal insulation shell was further modified accordingly to achieve pre-specified cooling rates, as demonstrated in the Results and Discussion section.

While the experimental protocol, a detailed mathematical analysis of the system, and uncertainty analysis have been previously presented [25], the key equations are represented here in brief, as they are important for interpretation of results. The analysis requires a pressure-volume calibration curve for the specific pressure tube, which was generated by substituting the cooling chamber with micro-syringe and recording pressure versus displacement data.

Since the cooling chamber is made of brass, which is a well-characterized material, the linear strain in the sample can be extracted as:

$$
\varepsilon_{i}=\frac{1}{3} \frac{\Delta V_{i}+\Delta V_{\text {brass }, i}}{V_{0}}
$$

where $V_{O}$ is the initial volume of the cooling chamber, $\Delta V_{i}$ is the volume change of the specimen, and $\Delta V_{\text {brass }, i}$ is the volume change of the brass chamber between its initial temperature, $T_{0}$, and a specific given temperature, $T_{i}$. The change in brass volume $\Delta V_{\text {brass, } i}$ is calculated by:

$$
\Delta V_{\text {brass }, i}=3 V_{0} \int_{T_{0}}^{T_{i}} \beta_{\text {brass }} d T
$$

where $\beta_{\text {brass }}$ is the thermal expansion property of brass.

The rate of change of the thermal strain with respect to temperature is defined as the thermophysical property of thermal expansion, $\beta$, which can be derivative from: 


$$
\beta=\frac{d \hat{e}}{d T}
$$

where $\hat{e}$ is a polynomial approximation of the thermal strain $\varepsilon_{i}$, at all measurement points. While the property of thermal expansion is intrinsic, the thermal strain is a relative measure specific to a particular process (i.e., the integral of thermal expansion between two relevant temperatures). Hence, the polynomial approximation $\hat{e}$ can be conveniently shifted for the purpose of analysis and/or presentation.

Equations (1)-(3) conveniently describe the analysis of thermal expansion for a chamber filled with a fluid (CPA-SIB cocktail in the current study). In order to analyze the thermal expansion of a CPA-permeated specimen, the experimental analysis must be performed in two consecutive steps: (i) measuring thermal expansion of the pure cocktail as described above, and (ii) measuring the thermal expansion of a chamber containing CPA-permeated tissue specimens, where the remainder of the chamber is filled with the same CPA under investigation. Once the ratio of specimen volume to chamber volume, $x$, is established, the thermal strain in the second step of experimentation is calculated by:

$$
\varepsilon_{i}=\frac{1}{3} \frac{\Delta V_{i}+\Delta V_{\text {brass }, i}}{V_{0}}=(1-x) \varepsilon_{\text {CPA }}+x \varepsilon_{\text {tissue }}
$$

where $\varepsilon_{C P A}$ is the thermal strain of the CPA solution as measured in the first step of experimentation. It follows that:

$$
\varepsilon_{t i s s u e}=\frac{\varepsilon_{i}-(1-x) \varepsilon_{C P A}}{x}
$$

\section{Materials Tested}

In previous studies along the current line of research, the following CPA cocktails have been tested: VS55, DP6, and DMSO in various applicable concentrations [24,26,27]. DP6 is a cocktail of $234.4 \mathrm{~g} / \mathrm{L}$ DMSO (3M), $228.3 \mathrm{~g} / \mathrm{L}$ propylene glycol (3M), and $2.4 \mathrm{~g} / \mathrm{L}$ HEPES in EuroCollins solution. VS55 is a cocktail of $242.14 \mathrm{~g} / \mathrm{L}$ DMSO $(3.1 \mathrm{M}), 168.38 \mathrm{~g} / \mathrm{L}$ propylene glycol (2.2M), $139.56 \mathrm{~g} / \mathrm{L}$ formamide (3.1M), and $2.4 \mathrm{~g} / \mathrm{L}$ HEPES in EuroCollins solution. The two cocktails are similar, excepting the exclusion of formamide from DP6 to reduce its toxicity. In return, DP6 contains a higher concentration of propylene glycol. While VS55 has been found to be a much better glass promoting cocktail [26,27], DP6 appears to be more promising due to its reduced overall CPA concentration, and therefore its toxicity.

DP6 with, or without additional ice modulators, has not yet been used extensively in biological cryopreservation principally because the focus to this point has been to characterize the physical properties and attributes of the formulation, which by design has a significantly lower total solute concentration than the baseline reference VS55 solution. It was also formulated to omit the formamide component of VS55, which has been proposed as an unnecessary and potentially toxic element of VS55 [7] that does little to promote the vitreous state. Some pilot studies comparing DP6 with VS55 for cryopreservation of pancreatic islets provide initial support for the hypothesis that DP6 will be less toxic by virtue of the lower total CPA concentration [41]. The downside in the application of DP6 is the high cooling rate typically required for vitrification. Hence, the current pilot study focuses on DP6 combined with SIMs, in an effort to improve its performance.

While many SIMs could be selected for the current pilot study, the choice of practice is based on recent experience developed by the current research team [40]. The following 
compounds were thus selected as representing the broad classification of ice-control molecules: $6 \%$ 1,3-cyclohexanediol (1,3-CHD), $6 \%$ 2,3 butanediol, and 12\% polyethylene glycol 400 (PEG400), which is a low molecular weight grade of polyethylene glycol.

Attempts to understand the requirements of vitrification solutions at the molecular level have led to identification of new solutes with physical properties that would promote the vitreous state during cooling. A promising class of compounds is the polyalcohols such as 1,2-propanediol (1,2PD), which is a component of DP6, and butane-2,3-diol (2,3BD), the optical isomers of which have been shown to vitrify at significantly lower concentrations than any other known CPA [3,36]. This is important to minimize the toxic effects of using high concentrations of CPAs. The use of 2,3 BD $(6 \%=0.7 \mathrm{M})$ as a supplemental SIM in this study is well within the toxic limits since we have previously published data showing tolerance of pancreatic islets exposed to up to $3 \mathrm{M} 2,3 \mathrm{BD}$ [39,41]. Moreover, it is now established that both the concentration needed to vitrify and the toxicity can be reduced by incorporating a wide variety of non-permeating disaccharides or polymeric compounds $[2,36]$; hence the selection of PEG400, which we have previously reported to elevate the glass transition temperature, reduce the energy associated with the transition, and reduce the risk of devitrification in a concentration dependent manner [4]. 1,3-CHD falls within the sub-classification of SIBs that are specifically designed to lattice-match with the basal plane of an ice crystal by hydrogen bonding and thereby inhibit ice crystal growth $[10,40]$.

The tissues models used in the current study are bovine muscles and goat carotid arteries. All tissue samples were donated by local slaughterhouses and no animals were sacrificed for the purpose of this study.

\section{RESULTS AND DISCUSSION}

Figure 2 displays a typical thermal history during experimentation, with a temperature difference of less than $3^{\circ} \mathrm{C}$ across the specimen. The small temperature difference and the exponential decay in chamber temperature indicate that the chamber can be treated as a lumped-capacity system, which is consistent with the mathematical formulation presented above. Hence, results in Fig. 2 indicate that modifying the cooling chamber to have a smaller volume and faster cooling rate did not affect system performance.

Figure 3 displays typical results for the pressure versus temperature curve, obtained in DP6 and DP6+12\% PEG400 experiments. Note that the pressure in the system is proportional to the overall volumetric contraction and therefore to the strain. With respect to the DP6 curve (upper curve), the material contracts nearly linearly with temperature in section A-B, exhibiting a simple fluid behavior during cooling. Volume expansion is evident in section $\mathrm{B}-\mathrm{C}$, indicating crystallization in the specimen. Due to the extent of crystallization, the system stopped functioning in sections $\mathrm{C}-\mathrm{D}$ and $\mathrm{D}-\mathrm{E}$, and the related output is meaningless. The material regains flow capability at point $\mathrm{E}$ during rewarming, where additional crystallization during rewarming is evident in section E-F; that is rewarming phase crystallization (RPC) in a partially vitrified material. The crystals start to melt at point F, a process which is completed by point $\mathrm{G}$. The material regains its simple fluid behavior in section $\mathrm{G}-\mathrm{H}$. The range between point $\mathrm{G}$ and point $\mathrm{B}$ is the range of super cooling. In sharp contrast, no crystallization or RPC are demonstrated for DP6+12\% PEG400 following the same thermal history. Here, the exceptional system response between point $\mathrm{B}$ and $\mathrm{G}$ is associated with the high level of viscosity and departing from a simple fluid-like behavior. Thermal expansion analysis in the current study is performed on section A-B of each curve.

Figure 4 presents the measured cooling rates from the experiments displayed in Fig. 3. It can be seen from Fig. 4 that the cooling rate in the temperature range of interest is close to 
linearly dependent upon temperature, which means that the thermal history can be approximated as a second order in time in the range of interest. The CPA cocktails were cooled at similar rates, ranging from $-4.5^{\circ} \mathrm{C} / \mathrm{min}$ at the beginning of cooling down to below $-1{ }^{\circ} \mathrm{C} / \mathrm{min}$ as the CPA cocktail approached $-100^{\circ} \mathrm{C}$. The rewarming phase followed a somewhat similar pattern. Interestingly, the effects of RPC are evident from the pure DP6 results, which support the observations of RPC followed by melting in the rewarming phase. It is noted that those rates of cooling and rewarming are considered very slow in the context of vitrification, which supports the effort to achieve large-scale vitrification in due course.

>Figures 5 - Figure 8 display experimental results, whereas Table 1 lists parametric estimation results for the corresponding thermal expansion coefficient. Figure 5 displays the effect of SIMs on thermal expansion of DP6 in the presence and absence of bovine muscle specimens. In the absence of tissue specimens at $-55^{\circ} \mathrm{C}$ for example (which is the onset of crystallization for pure DP6 at the corresponding thermal history, Fig. 4), the thermal strain increases by $8 \%$ to $11 \%$ when the different SIMs are added, with $12 \%$ PEG400 displaying the most significant effect. Suppression of crystallization is evident when SIBs are combined, as the minimum temperature achieved (point B on Fig. 3) has been significantly lowered.

In order to demonstrate repeatability and consistency, Fig. 6 presents all experimental data used to approximate the polynomials displayed in Fig. 5, for experiments in the absence of tissue specimens. In general, data scattering for DP6+12\%PEG400 is at a narrower range than that found for DP6 combined with the other two SIMs. In fact, it simply appears as one would expect from a cocktail with a higher concentration than DP6. The effects of 6\% 1,3CHD and 6\% 2,3 butanediol are expressed in increased data scattering, which is likely to be associated with the stochastic nature of ice nucleation and the SIM interference with crystal growth. Nevertheless, the absolute volume fraction that this strain scattering represents is less than $0.25 \%$ (i.e., $=0.0025$ ). While such a small volume represents insignificant portion of crystals, if indeed the scattering is directly related to crystallization, such a variation in strain may be quite significant in solid mechanics analysis. In general, while a ductile material, such as the tissue above freezing temperatures, may sustain strains of the order of $1 \%$, strains of a smaller order may lead to fracture formation in a brittle material such as glass. One example of generating such strains would be when cooling of an organ, where its outer portion vitrifies first due to a radial process of heat transfer, which results in applied forces on the inner-warmer region. In such a case, the outer surface would experience tensile circumferential stresses, which can potentially lead to fracture propagation from the surface inwards.

With regard to the bovine muscle experiments displayed in Fig. 5, CPA-SIM cocktail permeation was achieved by immersion of approximately $1.5 \mathrm{ml}$ of tissue into $3 \mathrm{ml}$ of full concentration solution in three steps. These steps are not optimized for tissue functionality nor viability, as the experimental protocol is aimed at physical testing only. In order to test the assumption that the solution fully equilibrates, bovine muscles permeated with DP6 + $12 \%$ PEG400 were immersed for varying time periods of 2, 4, 8, 12, 24, and 48 hours, with each step taking up to a third of the complete immersion time. Based on those tests and for data analysis based on the cooling portion of the curve (Section A-B in Fig. 3), there appears to be no correlation between the immersion time and the measured thermal expansion. While not relevant to thermal expansion measurements in the current study, RPC was evident for shorter immersion times for bovine muscles permeated with both DP6 + $12 \%$ PEG400 and DP6 + 6\% 1,3-CHD. Regardless of permeation time, RPC was always evident in experiments performed on bovine muscles permeated with DP6 + 6\% 2,3 butanediol. In general, the critical rewarming rate to prevent crystallization is higher in magnitude than the critical cooling rate to prevent crystallization in the process of 
vitrification. However, the specific cooling rates and the kinetics of SIB permeation are yet to be explored by means of DSC and spectroscopy, respectively. Interesting to note that in the pure DP6 experiments in the current study, Unisol was replaced with EuroCollins as a buffer solution, which had no effect on thermal expansion results, as compared with a prior study [24].

It can be seen from Fig. 5 that permeated tissue specimens contract less than the pure cocktails, which is consistent with previous studies in the absence of SIMs [24]. However, lower minimum temperatures were achieved in the current study, due to the particular combinations of CPA and SIMs. Such a differential expansion between the tissue and the surrounding solution has implications on the design of packaging for vitrified materials, in effort to reduce resulting fractures. It has been shown previously that such a difference may result in fracture initiation in the surrounding solution [35]. Once fractures are triggered, their propagation requires smaller driving forces, and it is difficult to predict where or by what fractures may be arrested. In general, a thermal expansion difference of up to $15.1 \%$ is observed between tissue permeated with a CPA-SIM cocktail and the thermal expansion of the pure cocktail.

Figure 7 displays results for goat artery samples, following a similar procedure to the one described above for bovine muscle samples. The thermal strain of DP6 + 12\% PEG400 in the absence of tissue samples is in a much closer range with permeated arteries (up to 3.5\% for a temperature range of $80^{\circ} \mathrm{C}$ ) than with permeated muscle specimens (up to $15.1 \%$ for a temperature range of $80^{\circ} \mathrm{C}$ ). Interestingly, the thermal strain of goat arteries permeated with $6 \% 1,3-\mathrm{CHD}$ is actually greater than that of the solution itself from an unknown reason at this stage (up to $6.5 \%$ for a temperature range of $80^{\circ} \mathrm{C}$ ) and is quite similar to that of goat arteries permeated with DP6 + 12\% PEG400. Finally, the thermal strain for goat arteries permeated with $6 \% 2,3$ butanediol showed less thermal strain than that of bovine muscles permeated with that solution (up to $8.7 \%$ for a temperature range of $80^{\circ} \mathrm{C}$ ). Overall, thermal strain in permeated goat arteries tended to be closer to that of the pure solution than to that of permeated bovine muscles. As indicated above, these differences may be associated with techniques and effects of permeation, with an optimal procedure remaining to be explored in future studies for tissue viability and functionality. Following a similar procedure to the one presented in [25], analysis of the current system indicates absolute uncertainty range of $8 \%$ for a single independent measurement with the experimental system, including systematic errors originated from calibration, geometrical dimensions, and the characteristics of the pressure transducer (gain, bias, repeatability, etc.). Taking into account that the above observations rely on differences between polynomial approximations based on datasets and not on single independent measurements, and taking into account that all datasets were obtained with the system after calibration, the above observations are deemed significant.

The current experiments are conducted at very low cooling rates and, therefore, are deemed relevant to scale-up efforts of cryopreservation by vitrification. In particular, the typical cooling rates displayed in Fig. 4 were found to be at the threshold for vitrification of goat artery specimens permeated with DP6 +6\% 1,3-CHD. Here, Fig. 8(c) displays results from ten experimental runs under identical conditions, where the results could be grouped into two groups of five: (i) crystallization is not evident - the strain curve is close to linear and scattering of results is in a very narrow range (results included in Table 1), and (ii) crystallization is evident-the strain curve is quadratic in nature and scattering of results is significant (results omitted from Table 1). The reason for smaller strains during crystallization is that cooling with no phase change is associated with contraction, while ice crystallization is associated with volume expansion of the pure water. These are competing effects, causing the overall strain to decrease. Since water crystallization is a dramatic effect of about 9\% volume increase, the relative small changes in the strain rate in Fig. 8(c) 
suggest partial vitrification only (more significant crystallization is shown in Section B-C of Fig. 3).

Figure 9 displays estimation for the extent of partial crystallization in Fig. 8(c). This estimation is based on the difference between the two best-fit curves for goat arteries permeated with DP6 + 6\% 1,3-CHD (for small strains, the volumetric strain is triple the linear strain displayed in Figs. 5-8). Note that uncertainty in strain measurements is estimated as $8 \%$, which is the strain difference between the different curves at $-30^{\circ} \mathrm{C}$, a point at which the extent of partial crystallization is estimated as 1.8\% (Fig. 9). Since the maximum value of partial crystallization presented in Fig. 9 is $15.5 \%$, partial crystallization is found to be a significant effect in this experimental set. Nevertheless, the shape and distribution of crystals in the system remains unknown at this stage. It is reemphasized that the SIMs selected for this study are considered not to prevent ice nucleation but to prevent the growth of crystals. The presence of nuclei or small portion of ice crystals is not necessary detrimental to the success of cryopreservation via vitrification, as has been demonstrated recently on blood vessels at marginal cooling rates [1].

\section{SUMMARY AND CONCLUSIONS}

Investigation of solid mechanics effects in cryopreservation represents a largely unchartered area of research. To the best of our knowledge, investigation of thermo-mechanical effects associated with the application of SIMs to vitrification has never been published before. The current study is focused on measuring the driving mechanism to thermo-mechanical stressthe physical property of thermal expansion. This study focuses on the upper part of the cryogenic temperature range, where the CPA behaves as a fluid for all practical applications. Concurrent efforts are devoted to measure thermal expansion at lower cryogenic temperatures, where the vitrified material behaves like a solid.

Results of this study demonstrate that the SIMs under investigation facilitate DP6 vitrification at low cooling rates in the range of 1 to $4{ }^{\circ} \mathrm{C} / \mathrm{min}$, which relaxes the constraints on large-scale cryopreservation. In fact, cooling pure DP6 alone always resulted in crystallization at some point in the process subject to the same thermal protocol. SIMs were found effective not only in suppressing crystallization but also in preventing RPC. It appears that the addition of SIM can potentially lead to two competing effects: (i) increased thermal expansion of the CPA-SIM cocktail, which may lead to higher thermo-mechanical stresses for a particular cooling rate, and (ii) decreased cooling/rewarming rates necessary to achieve a high degree of vitrification. Nevertheless, the magnitude of each effect will be dependent upon the specific setup and geometry of the problem and, thus, it is impossible to generate general rules on which effect will be more significant.

The promising results of this study suggest the additional resources should be devoted to study the application of SIM to vitrification, including, tissue viability and functionally, kinetics of CPA-SIM permeation, and other thermo-physical properties of the CPA-SIMtissue system.

\section{Acknowledgments}

This project has been supported by Award Number R21EB009370 from the National Institute of Biomedical Imaging and Bioengineering. The content is solely the responsibility of the authors and does not necessarily represent the official views of the National Institute of Biomedical Imaging and Bioengineering or the National Institutes of Health 


\section{REFERENCES}

1. Baicu S, Taylor MJ, Chen Z, Rabin Y. Cryopreservation of carotid artery segments via vitrification subject to marginal thermal conditions: Correlation of freezing visualization with functional recovery. Cryobiology. 2008; 58:1-8. [PubMed: 19007768]

2. Boutron P, Peyrdieu JF. Reduction in toxicity for red blood cells in buffered solutions containing high concentrations of 2,3-butanediol by trehalose, sucrose, sorbitol, or mannitol. Cryobiology. 1994; 31:367-373. [PubMed: 7924394]

3. Boutron P. Levo- and dextro-2,3 butanediol and their racemic mixture: very efficient solutes for vitrification. Cryobiology. 1990; 27:55-69.

4. Brockbank KGM, Walsh JR, Song YC, Taylor MJ. Vitrification. Encyclopedia of Biomaterials and Biomedical Engineering. Chap. 292. Informa Healthcare. 2008:3046-3057. http:// informahealthcare.com/doi/abs/10.3109/9781420078039.292.

5. Brockbank, KGM.; Taylor, MJ. Tissue Preservation. In: Baust, JG.; Baust, JM., editors. Advances in Biopreservation. Boca Raton: CRC Press; 2007. p. 157-196.

6. Brockbank, KGM.; Schenke-Layland, K.; Greene, E.; Chen, Z.; Fritze, O.; Schleicher, M.; Kaulitz, R.; Riemann, I.; Fend, F.; Albes, J.; Stock, UA.; Lisy, M. Ice-free cryopreservation of heart valve allografts: better extracellular matrix preservation in vivo and preclinical results. Cell Tissue Bank. 2012. http://www.ncbi.nlm.nih.gov/pubmed/22212702

7. Campbell LH, Rutledge RN, Taylor MJ, Brockbank KGM. Evaluation of the relative cytotoxicity of the components of a vitrification solution in a variety of cardiovascular cells. Cryobiology. 1999; 39(4):362.

8. Fahy GM, Levy D, Ali SE. Some emerging principles underlying the physical properties, biological actions, and utility of vitrification solutions. Cryobiology. 1987; 24:196-213. [PubMed: 3595164]

9. Fahy, GM. Biological effects of vitrification and devitrification. In: Pegg, DE.; Karow, AM., Jr, editors. Biophysics of Organ Cryopreservation. New York: Plenum; 1987. p. 265-297.

10. Fahy, GM. Methods of using ice-controlling molecules. US Patent number. 6773877. 2004.

11. Fong LP, Hunt CJ, Taylor MJ, Pegg DE. Cryopreservation of rabbit corneas: assessment by microscopy and transplantation. Br. J. Ophthalmol. 1986; 70(10):751-760. [PubMed: 3535871]

12. Hunt CJ. Cryopreservation of human stem cells for clinical application: a review. Transfusion Medicine and Hemotherapy. 2011; 38:107-123. [PubMed: 21566712]

13. Hunt CJ, Taylor MJ, Pegg DE. Freeze substitution and isothermal freeze fixation studies to elucidate the pattern of ice formation on smooth muscle at $252 \mathrm{~K}\left(-21^{\circ} \mathrm{C}\right)$. J. Microsc. 1982; 125:177-186. [PubMed: 7086882]

14. Jacobsen IA, Pegg DE, Starklint H, Chemnitz J, Hunt C, Barfort P, Diaper MP. Effect of cooling rate and warming rate on glycerolized rabbit kidneys. Cryobiology. 1984; 21:637-653. [PubMed: 6394215]

15. Jimenez-Rios JL, Rabin Y. Thermal expansion of blood vessels in low cryogenic temperatures. Part I: A new experimental device. Cryobiology. 2006; 52(2):269-283. [PubMed: 16487503]

16. Jimenez-Rios JL, Rabin Y. Thermal expansion of blood vessels in low cryogenic temperatures. Part II: Measurements of blood vessels vitrified with VS55, DP6, and 7.05M DMSO. Cryobiology. 2006; 52(2):284-294. [PubMed: 16488407]

17. Jimenez-Rios JL, Steif PS, Rabin Y. Stress-strain measurements and viscoelastic response of blood vessels cryopreserved by vitrification. Annals of Biomedical Engineering. 2007; 35(12):20772086. [PubMed: 17828592]

18. Jimenez-Rios JL, Rabin Y. A new device for mechanical testing of blood vessels at cryogenic temperatures. Journal of Experimental Mechanics. 2007; 47:337-346.

19. Karow, AM. Biophysical and chemical considerations in cryopreservation. In: Karow, AM.; Pegg, DE., editors. Organ preservation for transplantation. New York: Dekker; 1981. p. 113

20. Luyet BJ. The Vitrification of Organic Colloids and of Protoplasm. Biodynamica. 1937; 1(29):114.

21. Mazur P. Freezing of living cells: mechanisms and implications. Am J Physiol. 1984; 247:125. 
22. Pegg, DE.; Jacobsen, IA.; Armitage, WJ.; Taylor, MJ. Mechanisms of cryoinjury in organs. In: Pegg, DE.; Jacobsen, IA., editors. Organ Preservation II. Edinburgh: Churchill Livingstone; 1979. p. 132-146.

23. Pegg, DE. Ice crystals in tissues and organs. In: Pegg, DE.; Karow, AM., editors. The Biophysics of Organ Preservation. New York: Plenum Publishing Corp; 1987. p. 117-140.

24. Plitz J, Rabin Y, Walsh J. The effect of thermal expansion of ingredients on the cocktails VS55 and DP6. Cell Preservation Technology. 2004; 2(3):215-226.

25. Rabin Y, Bell E. Thermal expansion measurements of cryoprotective agents. Part I: A new experimental apparatus. Cryobiology. 2003; 46(3):254-263. [PubMed: 12818215]

26. Rabin Y, Plitz J. Thermal expansion of blood vessels and muscle specimens permeated with DMSO, DP6, and VS55 in cryogenic temperatures. Annals of Biomedical Engineering. 2005; 33(9):1213-1228. [PubMed: 16133928]

27. Rabin Y, Taylor MJ, Walsh JR, Baicu S, Steif PS. Cryomacroscopy of vitrification, Part I: A prototype and experimental observations on the cocktails VS55 and DP6. Cell Preservation Technology. 2005; 3(3):169-183. [PubMed: 16721425]

28. Rabin, Y.; Steif, PS. Solid mechanics aspect of cryobiology. In: Baust, JG.; Baust, JM., editors. Advances in Biopresevation. Boca Raton: CRC Press; 2006. p. 359-382.

29. Rowley SD. Hematopoietic stem cell cryopreservation: a review of current techniques. J Hematother. 1992; 1(3):233-250. [PubMed: 1365030]

30. Scholtze, H. Glass: nature, structure, and properties. New York: Springer-Verlag; 1991.

31. Steif PS, Palastro M, Wen CR, Baicu S, Taylor MJ, Rabin Y. Cryomacroscopy of vitrification, Part II: Experimental observations and analysis of fracture formation in vitrified VS55 and DP6. Cell Preservation Technology. 2005; 3(3):184-200. [PubMed: 16900261]

32. Steif PS, Palastro MC, Rabin Y. Analysis of the effect of partial vitrification on stress development in cryopreserved blood vessels. Medical Engineering \& Physics. 2007; 29(6):661-670. [PubMed: 16996295]

33. Steif PS, Palastro MC, Rabin Y. The effect of temperature gradients on stress development during cryopreservation via vitrification. Cell Preservation Technology. 2007; 5(2):104-115. [PubMed: 18185851]

34. Steif PS, Palastro MC, Rabin Y. Continuum mechanics analysis of fracture progression in the vitrified cryoprotective agent DP6. ASME Biomechanical Engineering. 2008; 130(2) 021006.

35. Steif PS, Noday DA, Rabin Y. Can Thermal expansion differences between cryopreserved tissue and cryoprotective agents alone cause cracking? CryoLetters. 2009; 30(6):414-421. [PubMed: 20309497]

36. Sutton RL. Critical cooling rates for aqueous cryoprotectants in the presence of sugars and polysaccharides. Cryobiology. 1992; 29:585-598. [PubMed: 1424715]

37. Taylor MJ, Pegg DE. The effect of ice formation on the function of smooth muscle tissue following storage at $-21^{\circ} \mathrm{C}$ and $-60^{\circ} \mathrm{C}$. Cryobiology. 1982; 20:36-40. [PubMed: 6831909]

38. Taylor, MJ. Sub-zero preservation and the prospect of long term storage of multicellular tissues and organs. In: Calne, RY., editor. Transplantation immunology: clinical and experimental. Oxford: Oxford University Press; 1984. p. 360-390.

39. Taylor MJ, Foreman J. Tolerance of isolated pancreatic islets to butane-2,3-diol at $0{ }^{\circ} \mathrm{C}$. Cryobiology. 1991; 28(6):566-567.

40. Taylor, MJ.; Song, YC.; Brockbank, KGM. Vitrification in tissue preservation: new developments. In: Fuller, BJ.; Lane, N.; Benson, E., editors. Life in the Frozen State Taylor and Francis Books. London: 2004. p. 603-642.

41. Taylor MJ, Baicu S. Review of vitreous islet cryopreservation: some practical issues and their resolution. Organogenesis. 2009; 5(3):155-166. [PubMed: 20046679]

42. Wowk B, Leitl E, Rasch CM, Mesbah-Karimi N, Harris SB, Fahy GM. Vitrification enhancement by synthetic ice blocking agents. Cryobiology. 2000; 40(3):228-236. [PubMed: 10860622] 


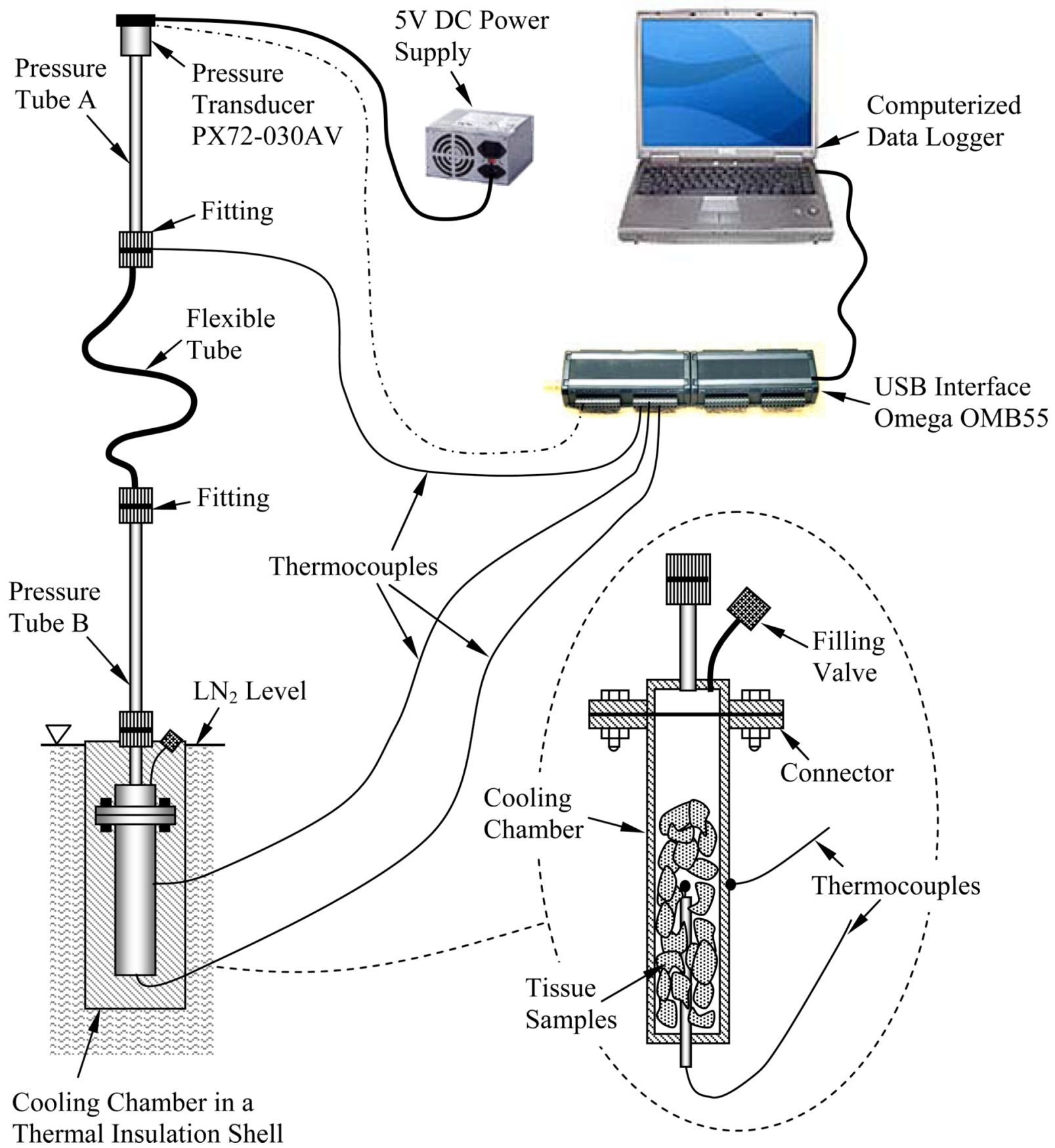

Figure 1.

Schematic illustration of the experimental apparatus [26] (with kind permission from Springer Science). 


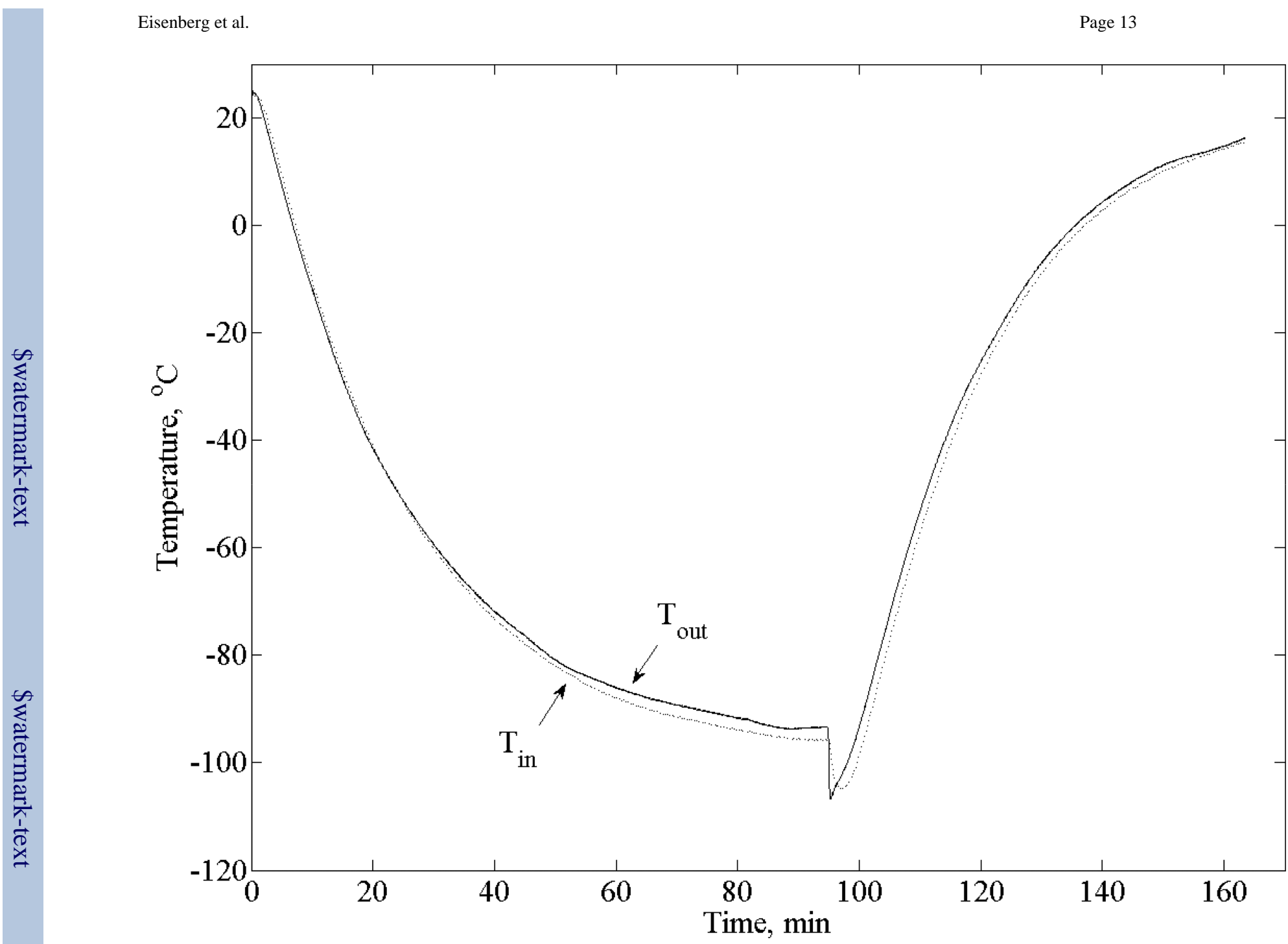

Figure 2.

Thermal history from a typical experiment, where $\mathrm{T}_{\text {in }}$ and $\mathrm{T}_{\text {out }}$ are the temperatures at center and the outer wall of the chamber, respectively. 


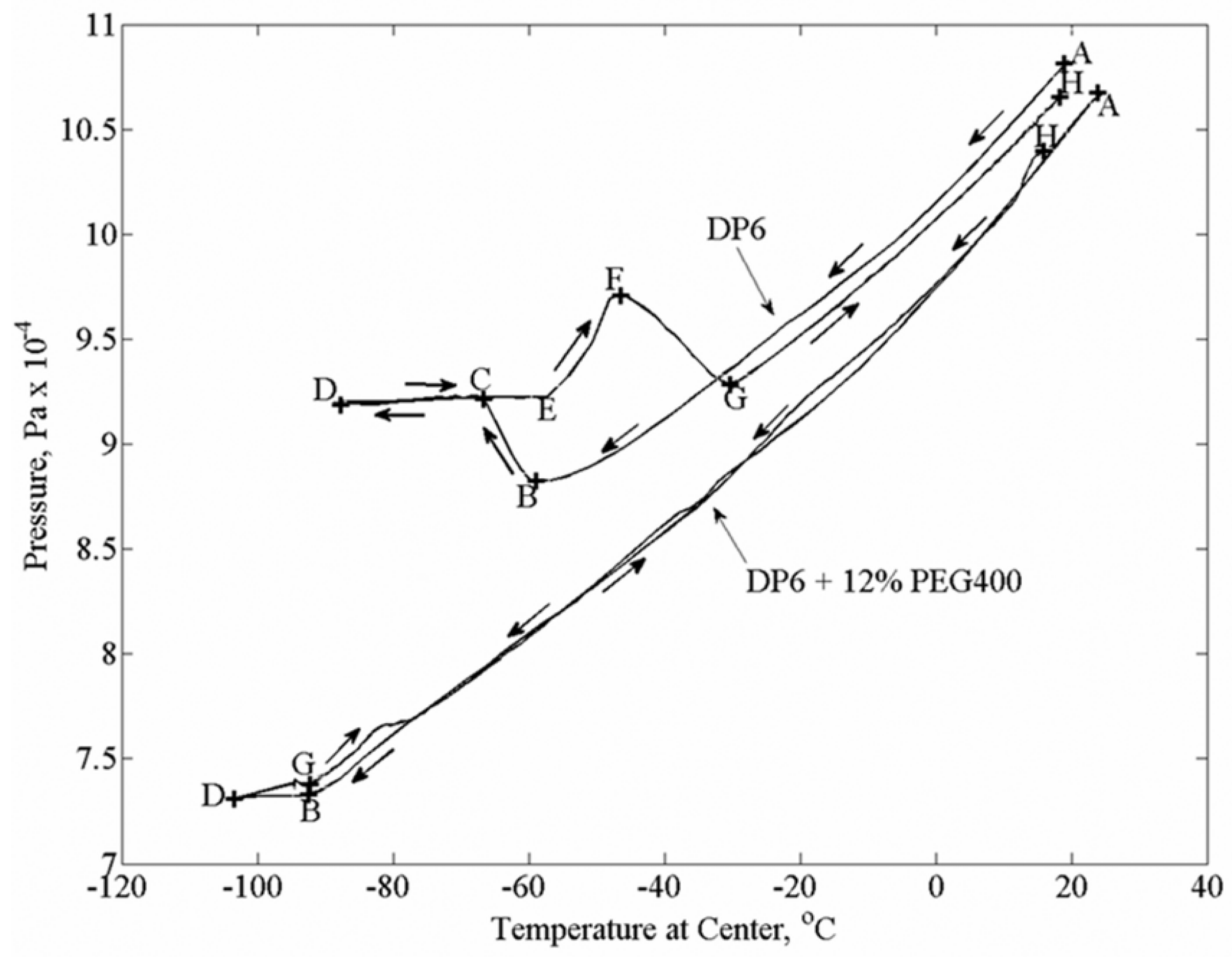

Figure 3.

Experimental results for pressure versus temperature for two typical cases: (i) DP6 without SIM, and (ii) DP6 + 12\% PEG400 (the letters represent key points in the process, as described in the text) 


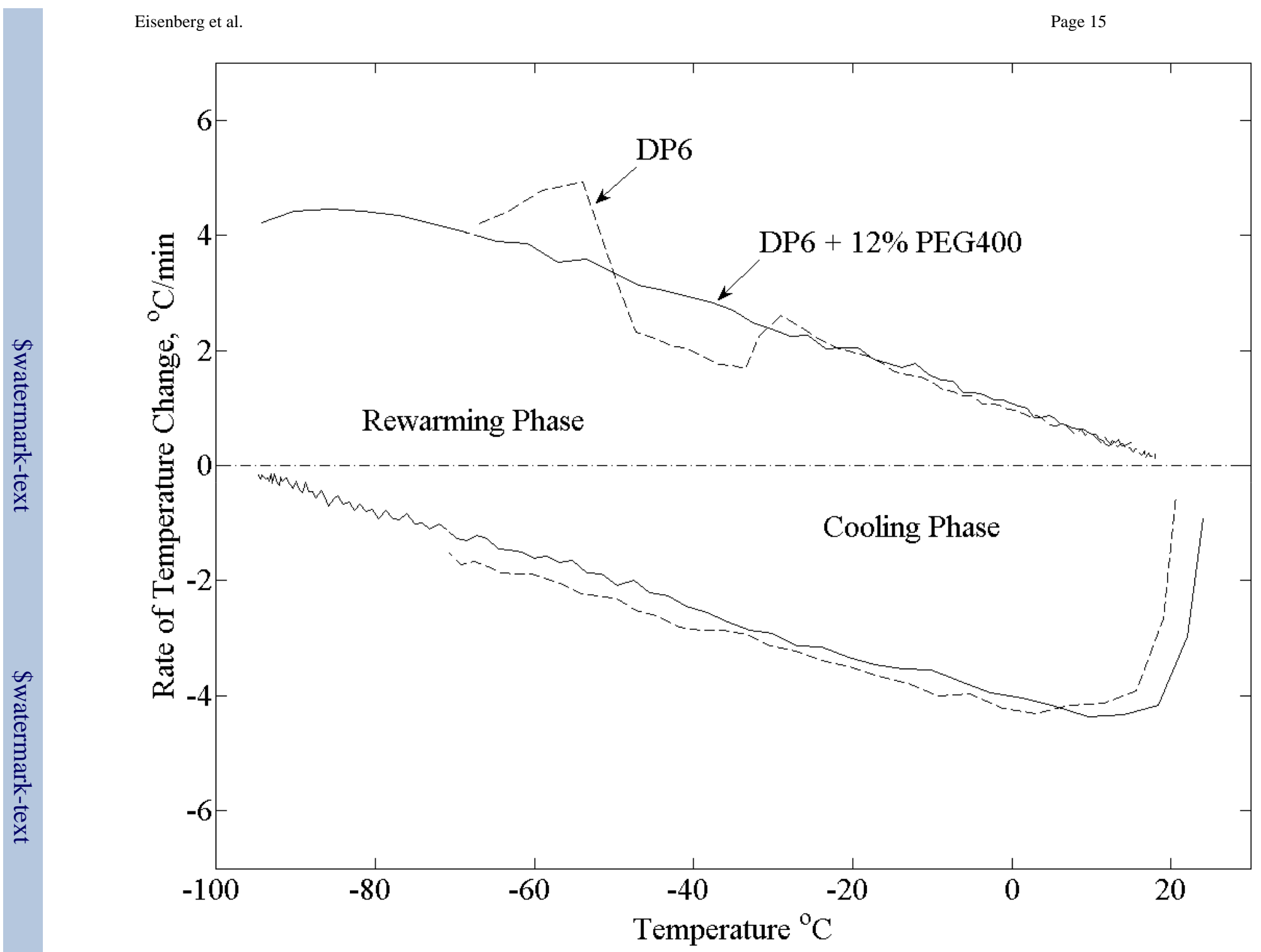

Figure 4.

Cooling and rewarming rates for the two experiments shown in Fig. 3 


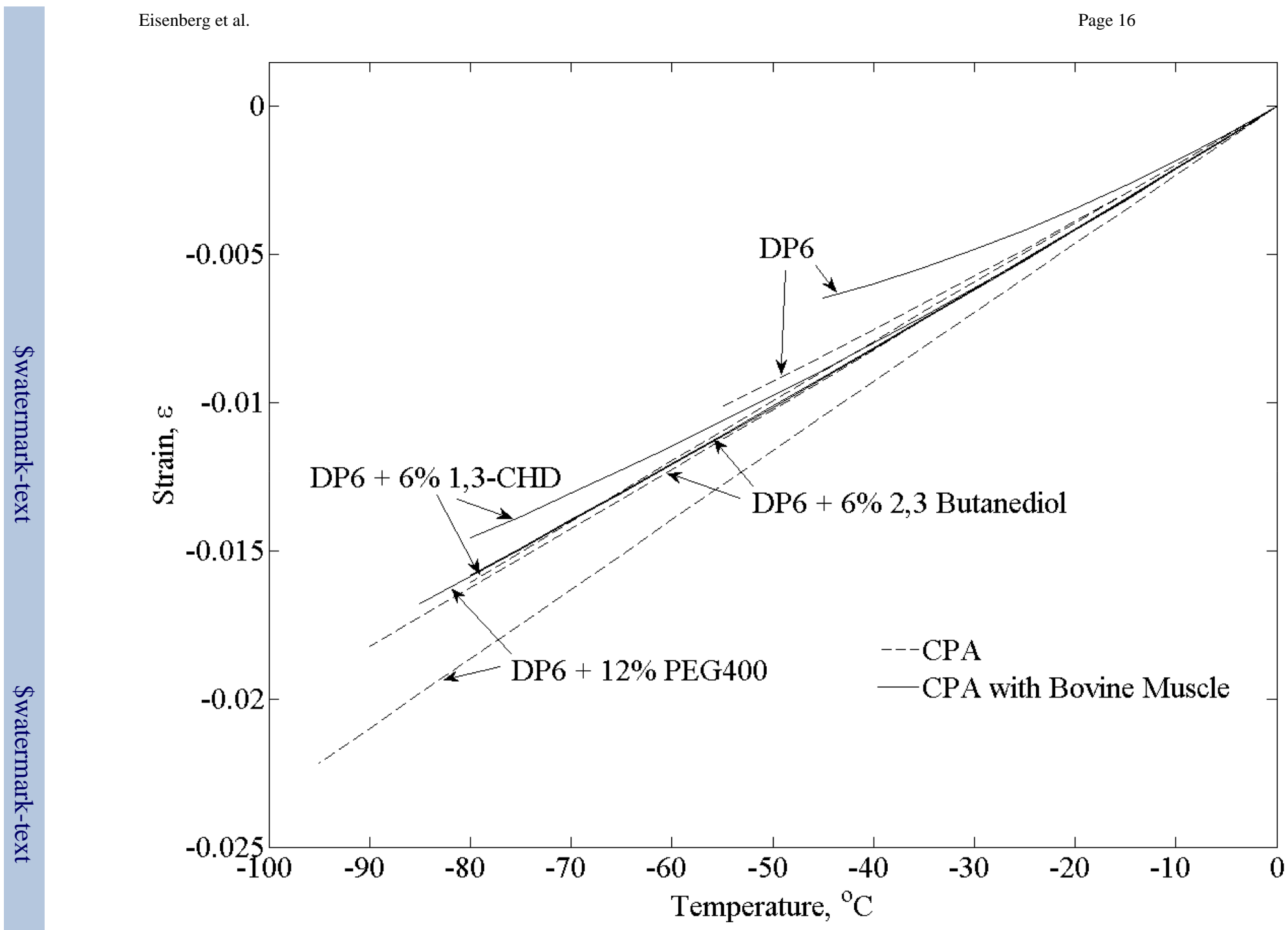

Figure 5.

Polynomial approximation of the thermal strain for DP6 combined with various SIMs in the presence and absence of bovine muscle specimens 

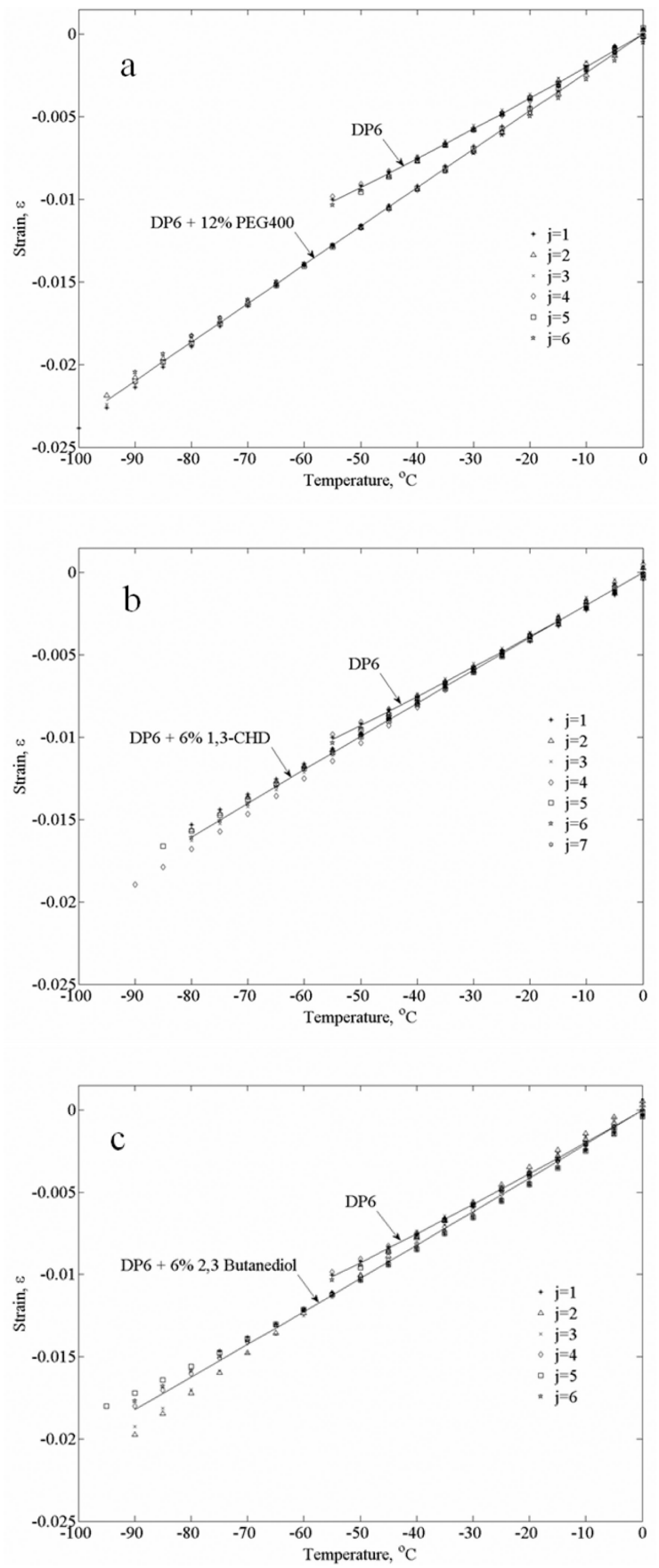

Figure 6.

Thermal strain data from all the individual experiments used for the polynomial approximations displayed in Fig. 5: (a) DP6+12\% PEG400 (b) DP6+6\% 1,3-CHD, and (c) DP6+6\% 2,3 butanediol; $j$ is the experiment number and solid lines represent the best-fit polynomial approximations. 


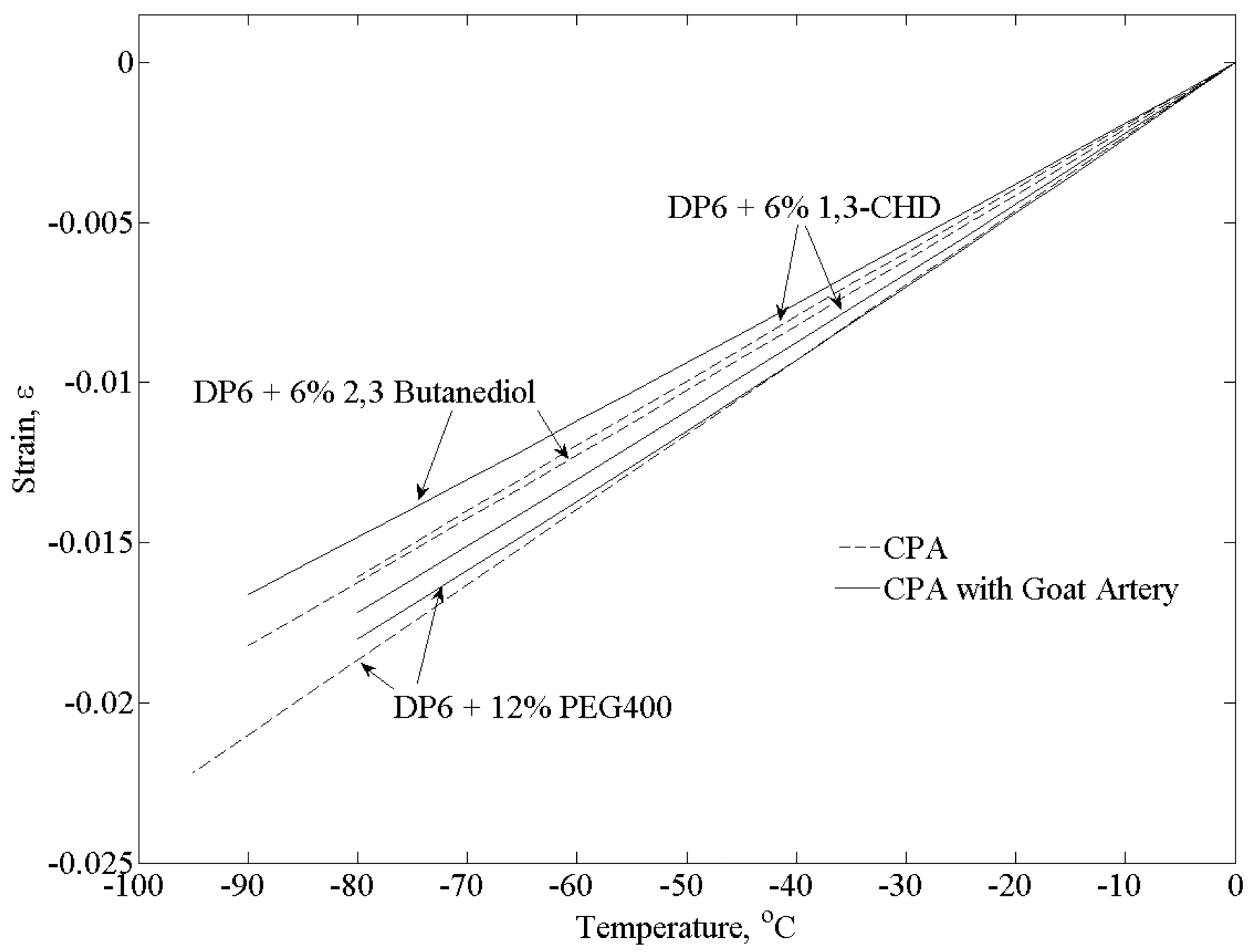

Figure 7.

Polynomial approximation of the thermal strain for DP6 combined with various SIMs in the presence and absence of goat artery specimens 

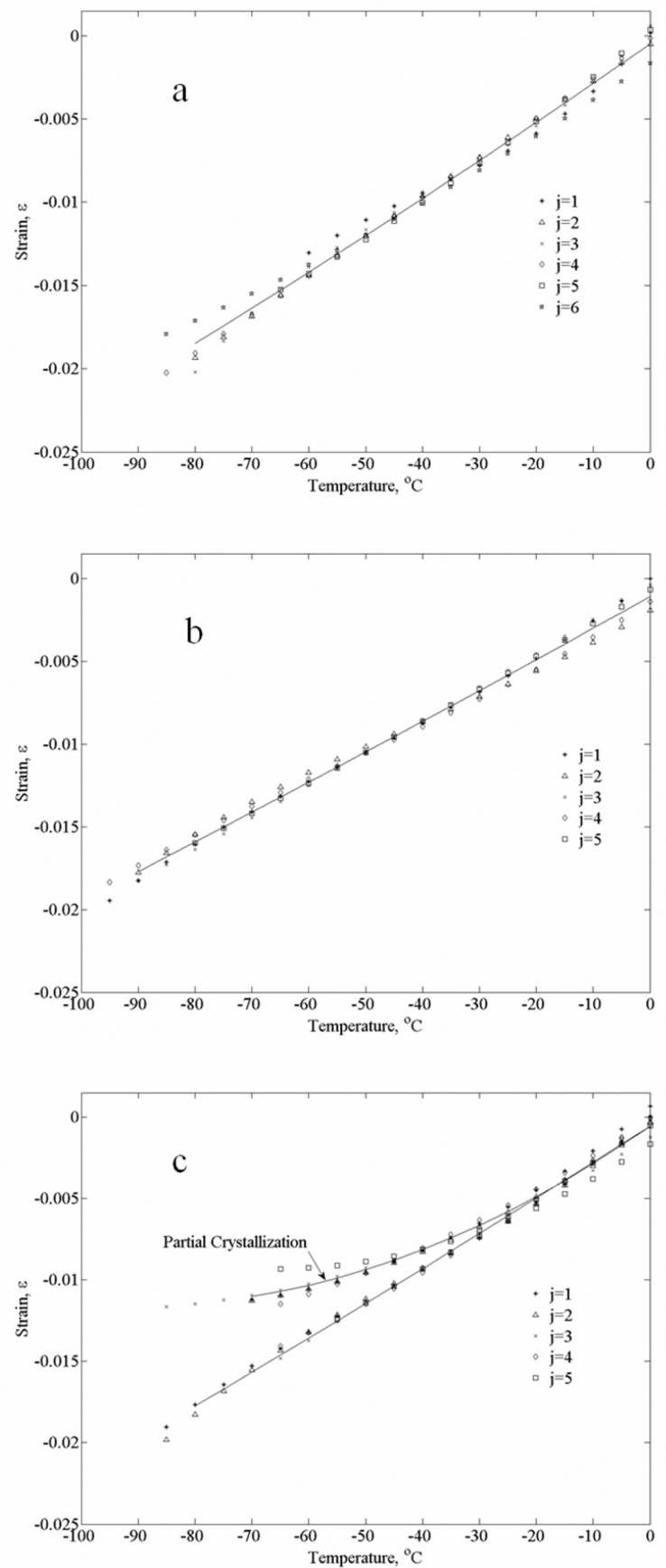

Figure 8.

Thermal strain data from all the individual experiments used for the polynomial approximations displayed in Fig. 7: (a) DP6+12\% PEG400 (b) DP6+6\% 1,3-CHD, and (c) DP $6+6 \% 2,3$ butanediol; $j$ is the experiment number and solid lines represent the best-fit polynomial approximations. 


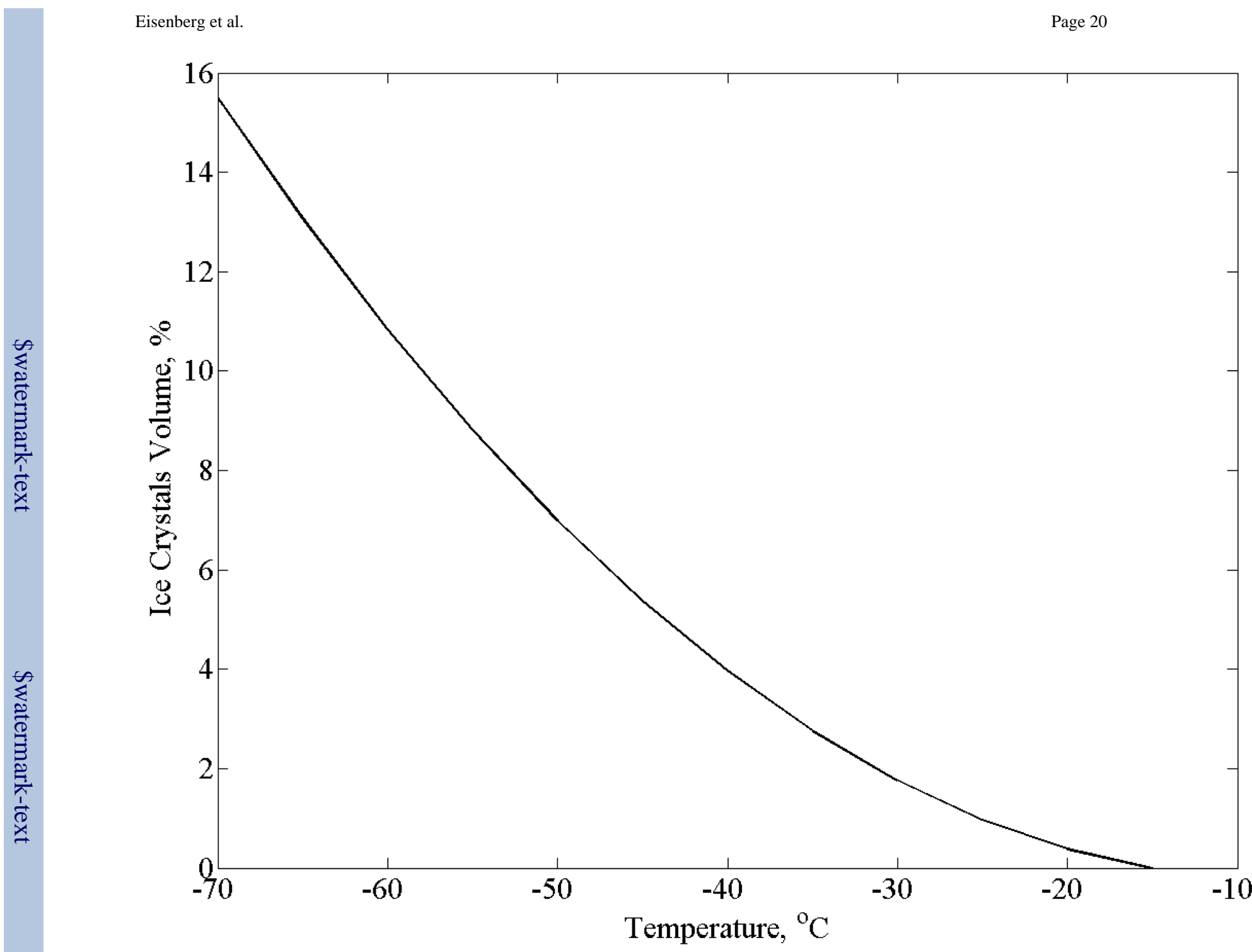

Figure 9.

Volume fraction of ice crystals in the case of partial vitrification displayed in Fig. 8(c): goat artery specimens permeated with DP6+6\% 1,3-CHD 


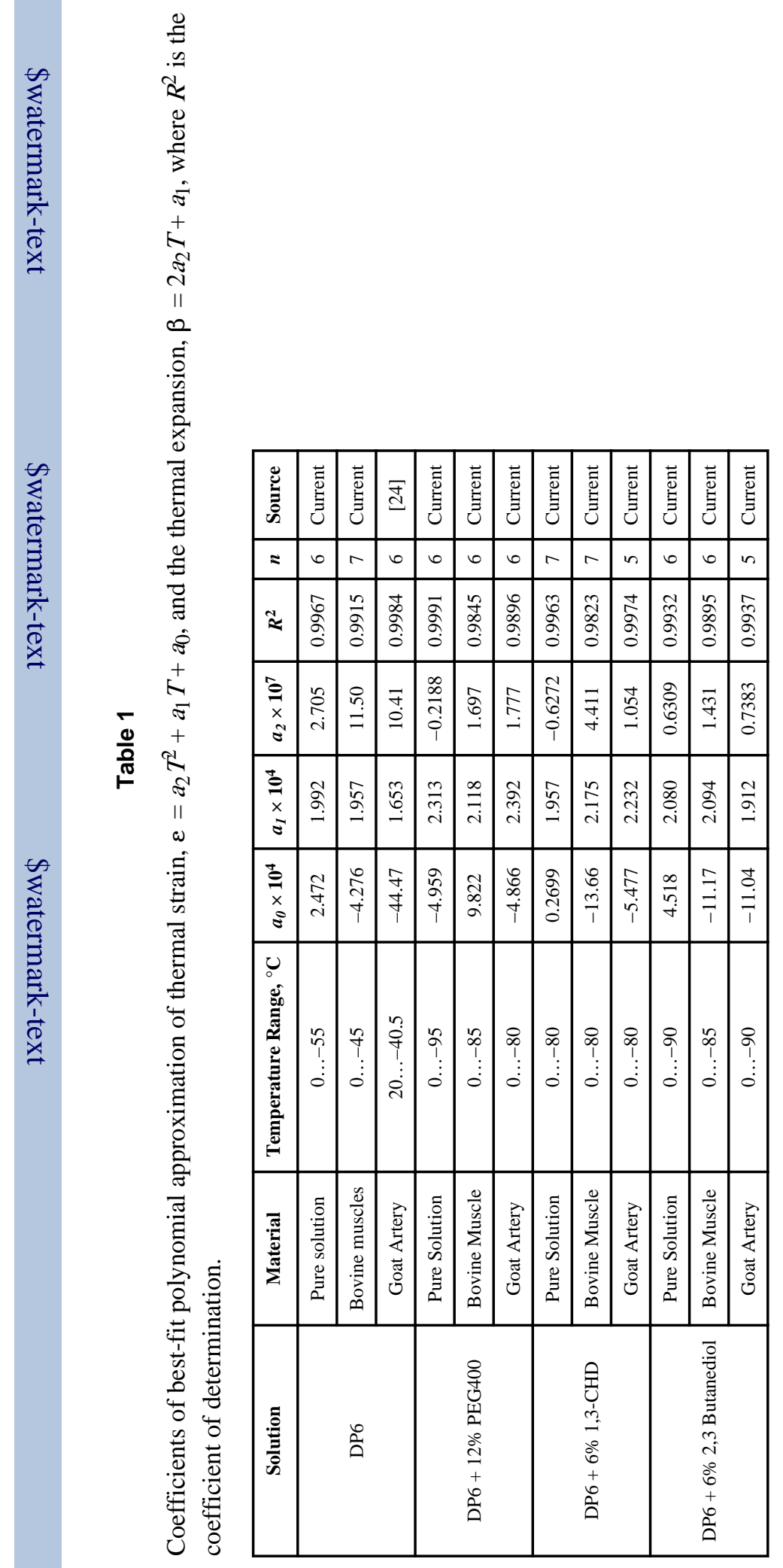

Cryobiology. Author manuscript; available in PMC 2013 October 01. 